\title{
Origins and Contrast of the Electron Signals at Low Accelerating Voltage and with Energy-Filtering in the FE-SEM for High Resolution Imaging
}

\author{
Hendrix Demers ${ }^{1}$, Nicolas Brodusch ${ }^{1}$, Patrick Woo $^{2}$, and Raynald Gauvin ${ }^{1}$ \\ 1. Department of Mining and Materials Engineering, McGill University, Montreal, Quebec, Canada. \\ 2. Hitachi High-Technologies Canada Inc., Toronto, Canada.
}

For developing new technologies, it is important to characterize materials at the nanoscale. To achieve high resolution, field emission scanning electron microscopes (FE-SEM) were developed. These microscopes allow working at low accelerating voltage, below $5 \mathrm{kV}$, to take advantage of the reduction of the interaction volume with accelerating voltage. Furthermore, their higher gun brightness compared to conventional thermo-electronic emitters [1], allow a probe size at the nanoscale. However, technical problems arise when SEM operates at low $\mathrm{kV}$ (source brightness decreases and chromatic aberration increases). Using the deceleration mode minimizes these problems. Further improvement is achieved by using a cold-field emitter, which has a smaller energy spread, a higher brightness, and a smaller probe size than a FE-SEM. At low accelerating voltage, the emission volume of the backscatter (BSE) and the secondary $\left(\mathrm{SE}_{\mathrm{II}}\right)$ electrons signals is close than the one of the $\mathrm{SE}_{\mathrm{I}}$ signals. Furthermore, the use of a magnetic field above the sample and probe deceleration improve the spatial resolution by collecting mostly high-resolution signals. In addition, the energy-filtering of the electron signals allows the selection of contrast detected: topographical, compositional, structural, or crystallographic.

Figure 1 and 2 are illustrations of high resolution and high contrast imaging at two different magnifications of a $\mathrm{Li}_{2} \mathrm{FeSiO}_{4}$ powder with two different in-lens electron detectors (upper and top) with a Hitachi SU8230 cold field-emission SEM. The powder was dispersed in a mixture of ethanol and ionic liquid, which provided charge compensation during imaging and allowed using the most suitable accelerating voltage $(2.2 \mathrm{kV})$ to obtain high contrast micrographs [2], to reduce beam spreading, and to increase the compositional contrast. In addition, energy-filtering was applied to the upper and top detectors. Both collected signals were composed of a combination SEs and BSEs, the upper detector collects low angle electrons whereas the top detector collects high angle electrons. In these micrographs, the upper detector signal was filtered to repulse the low energy SEs from the collector which improve the topographical contrast (Fig. 1A and 2A). In these micrographs, the signal is majority composed of high energy SEs (> 9 $\mathrm{eV}$ ) and low angle BSEs and the contrast is obviously topographic with a compositional component. On the other hand, the top detector was filtered at $80 \%$ leading to a signal composed mostly of high energy BSEs (1.76 to $2.20 \mathrm{keV}$ ). A high compositional contrast between iron oxide (bright) and ortho-silicate (dark grey) particles was observed with similar high spatial resolution. The high angle BSEs, collected by the top detector, are assumed to come from a small volume around the probe impact point. In addition, high energy BSEs have smaller emission volume, which further improve the spatial resolution. However, the energy-filtering and the collection solid angle of the top detector decrease the signal-to-noise ratio (SNR) of the micrographs (Fig. 1B and 2B).

Modern FE-SEMs, like the HITACHI SU-8230, provide low accelerating voltage, deceleration mode, and energy-filtering of the electron signals to allow the characterization of materials at the nanoscale with various types of contrasts. The understanding of the origins of the collected signals and effect of energyfiltering on electron micrograph contrast will extend the imaging capabilities of the FE-SEM towards new nanoscale applications. 
References:

[1] J.I. Goldstein et al, "Scanning Electron Microscopy and X-Ray Microanalysis", (Springer, 3rd edition, 2003).

[2] N. Brodusch et al, Microscopy and Microanalysis, 20 (2014), pp.38-39.

[3] D.C. Joy, Journal of Microscopy, 208 (2002), pp. 24-34.

[4] J. Kim et al, Journal of Vacuum Science Technology B, 25 (2007), pp. 1771-1775.
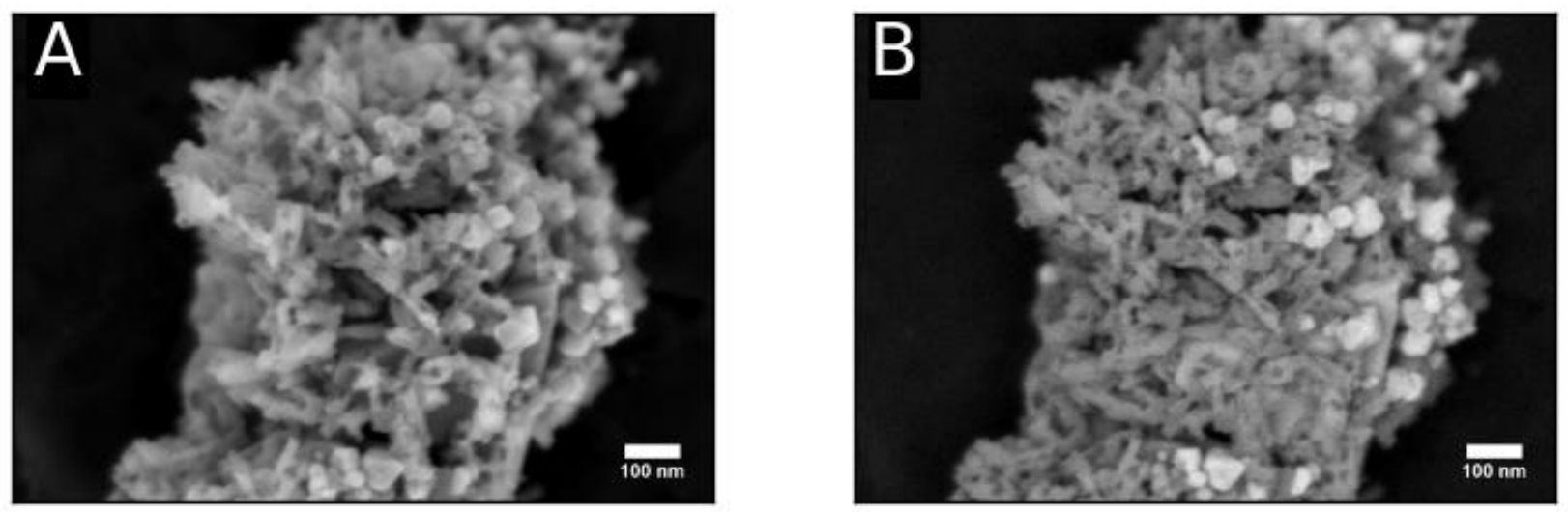

Figure 1. Electron micrographs of a $\mathrm{Li}_{2} \mathrm{FeSiO}_{4}$ lithium ortho-silicate powder with energy-filtering $\left(\mathrm{V}_{0}=\right.$ $2.2 \mathrm{kV}$ ) and ionic liquid preparation. A: Upper detector (filter bias $=9 \mathrm{~V}$ ): a resolution of $5.1 \mathrm{~nm}$ and a SNR of 65 were measured with SMART-J [3,4]. B: Top detector with filtering $(80 \%)$ : a resolution of $7.1 \mathrm{~nm}$ and a SNR of 2.9 were measured with SMART-J [3,4].
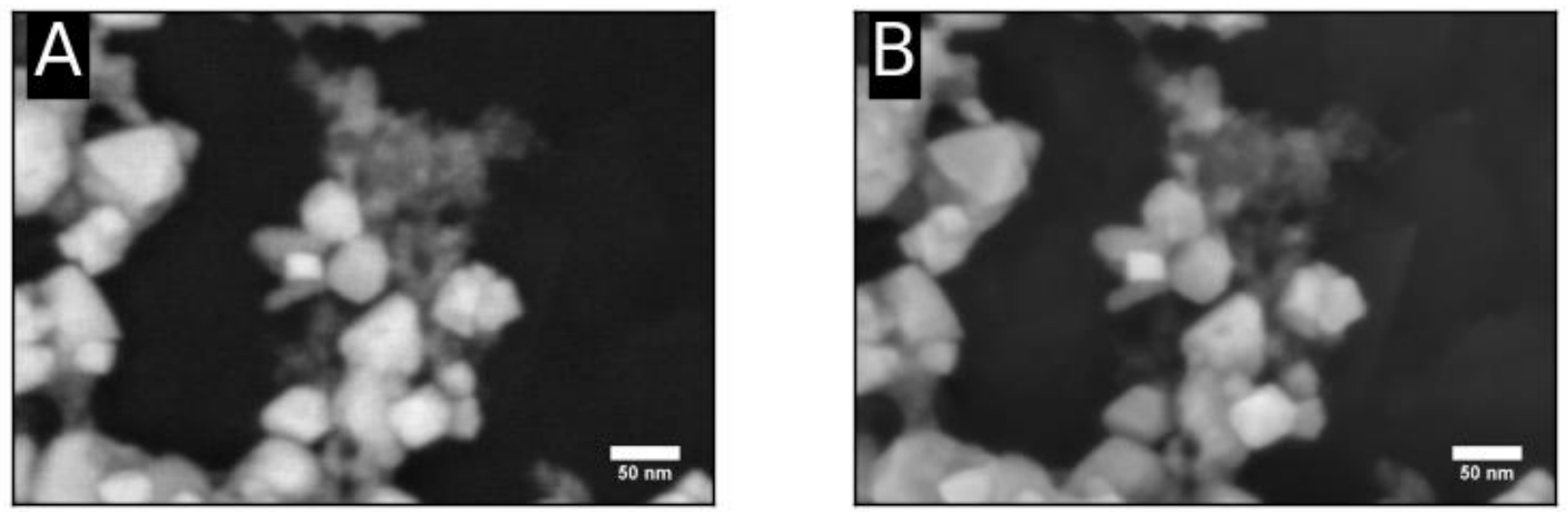

Figure 2. Higher magnification electron micrographs of $\mathrm{Li}_{2} \mathrm{FeSiO}_{4}$ lithium ortho-silicate powder with energy-filtering $\left(\mathrm{V}_{0}=2.2 \mathrm{kV}\right)$ and ionic liquid preparation. A: Upper detector (filter bias $=9 \mathrm{~V}$ ): a resolution of $4.7 \mathrm{~nm}$ and a SNR of 2.9 were measured with SMART-J [3,4]. B: Top detector with filtering (80\%): a resolution of $5.0 \mathrm{~nm}$ and a SNR of 0.5 were measured with SMART-J [3,4]. 